hoff have been followed especially in the Soviet Union by a host of high level mathematicians. Two directions at least have received particular emphasis in the USSR: the problems centering around stability-the Liapunov school, and the problems of asymptotic behavior. These are the precise topics to which Cesari's monograph is dedicated.

The Editors of the Ergebnisse Series have presented the author with a well nigh impossible task: to give a resume in a rather short space of so many lively contributions, a large part in Russian! Suffice to say that the author could not have acquitted himself better of the task: his little volume, distinguished by great carefulness and precision, is the guide par excellence in the labyrinth of recent work on ordinary differential equations. An important and additional merit is that it addresses itself not only to pure mathematicians but also to applied mathematicians and even to engineers. For instance, no little space is devoted to servo mechanisms and controls. Of great value also is a most extensive bibliography covering 60 pages! The Russian references are particularly abundant-a no mean advantage in view of their scattering and of the language barrier.

It is not profitable to give a detailed account of the very rich content of this Monograph, especially since, by and large, it is what one should expect. However, one may note more especially the following less expected features-no doubt a reflection of the reviewer's own taste and curiosity - a very careful treatment of Liapunov's stability theory and of his basic theorems, likewise of his type numbers; the inversion of his stability theorems by a number of authors mostly in the USSR but with Jose Massera in the lead; an extensive account of the author on his so-called $L$-diagonal process, and of Cesari and associates on periodic solutions of linear and nonlinear systems; the topological contributions of Waszewski and his group; the approximation methods of van der Pol and Krylov-Bogoliubov; the recent work on second order equations by Littlewood, Cartwright, by Levinson and by Levinson-Smith; the turning point theory of Langer; the work on asymptotic series by Wasow and by Turrittin.

In brief we have here a very careful survey of the work done on ordinary differential equations in the last few decades. The monograph is an invaluable source book on these activities and it will take a good deal of time before it is superseded.

\title{
S. LEFSCHETZ
}

Advanced calculus. By H. K. Nickerson, D. C. Spencer and N. E. Steenrod. Princeton, D. Van Nostrand Co., Inc., 1959. pp. ix +540, lithoprinted, $\$ 6.50$. 
Advanced Calculus is an ambiguous term which can include any or all of a large selection of topics. This book is a radical departure from all previous concepts of "Advanced Calculus," and the nature of this departure merits serious study of the book by everyone interested in undergraduate education in mathematics. The nearest "classical" description of the content of the book is that it is concerned with Vector Analysis and all the fringe topics that go with this, but no one should get the idea that this is a standard treatment of Vector Analysis.

The usual treatments of Vector Analysis have never found favor with the reviewer on two counts: (1) the "vectors" considered there are only indirectly related to the "vectors" of abstract algebra, and (2) the analysis is often presented in an intuitive fashion which avoids the major problems. For these reasons classical Vector Analysis has not been part of the main stream of mathematics and has become a special jargon favored by non-mathematicians. It is especially pleasing, therefore, to find that the authors of this book have rethought the whole subject of Vector Analysis and recast it so that it makes sense in the framework of contemporary mathematics.

In view of this introduction, no one will be surprised to find that the first chapter deals with the Algebra of vector spaces and that the second chapter treats Linear transformations of vector spaces. These chapters are relatively brief summaries of the essential definitions and theorems together with the necessary proofs. Although the presentation starts from the beginning, considerable maturity is assumed on the part of the student and previous acquaintance with Linear Algebra would definitely be helpful. The third chapter presents the Scalar product. This is done axiomatically, and then orthogonal bases are introduced and the formula for the scalar product in $R^{n}$ is developed.

Vector products in $R^{3}$ are introduced in the fourth chapter. Assuming that a scalar product is defined in a 3-dimensional vector space, the vector product is characterized by a set of axioms. It is then shown that two vector products exist which satisfy these axioms and that these differ only in algebraic sign. The usual formulas for triple vector and scalar products are developed.

The algebra of endomorphisms of a finite vector space with a scalar product is the subject of the fifth chapter. For 3-dimensional vector spaces of this kind, the determinant of an endomorphism is defined as the ratio of the scalar triple product of three transformed vectors to the scalar triple product of the three given vectors. Proper vectors and the characteristic polynomial of an endomorphism are defined. 
The adjoint of an endomorphism is defined abstractly in a fashion such that relative to a basis the matrix of the adjoint is the transpose of the matrix of the given endomorphism. This permits the definitions of the symmetric and skew-symmetric parts of an endomorphism. In particular it is proved that if $T$ is a skew-symmetric endomorphism and $X$ is a vector in the given 3 -dimensional vector space, then there exists a vector $A$ in the space such that $T X=A \times X$ for all $X$.

After five chapters of algebraic preparation, we start to find calculus in the sixth chapter which is titled Vector-valued functions of a scalar. This contains the standard material on limits, continuity, and derivatives, together with applications to arc-length, acceleration, steady flows, differential equations, and planetary motion. All functions are assumed to be differentiable, so that the analysis proceeds with a minimum of complications.

The plot thickens as we approach Scalar-valued functions of a vector in Chapter 7. This is what the old-timers would have called "functions of $n$-variables." The derivative of such a function is defined to be a scalar in the following sense due to Frechet:

Let $D$ be an open set in a finite dimensional vector space $V$, and let $R$ be the reals. The function $f: D \rightarrow R$ is the function under consideration. Let $X \in D$ and $Y \in V$; then by definition

$$
f^{\prime}(X, Y)=\lim _{h \rightarrow 0} \frac{f(X+h Y)-f(X)}{h}
$$

and this is called "the derivative of $f$ at $X$ with respect to $Y$."

In terms of this derivative, the gradient of $F, \nabla f$ is defined by the equation

$$
f^{\prime}(X, Y)=\nabla f(X) \cdot Y .
$$

Now it is possible to speak about directional derivatives and level surfaces. Line integrals are introduced and the usual theorems concerning gradients and the independence of a line integral relative to its path are proved.

For Vector-valued functions of a vector (Chapter 8), $F: D \rightarrow W$, the Frechet derivative $F^{\prime}(X, Y)$ is defined in a fashion similar to that above. In terms of bases for $V$ and $W$ this leads to the Jacobian matrix of $F$. When $V=W$ this derivative at a point of $D$ is an endomorphism of $V$. The trace of this endomorphism is defined to be the "divergence of $F$." The endomorphism has a skew-symmetric part $F_{-}^{\prime}(X, Y)$, and for 3-dimensional vector spaces the "curl of $F$ at $X$ " is defined by the equation: 


$$
2 F_{-}^{\prime}(X, Y)=\operatorname{curl} F(X) \times Y .
$$

It is shown that the divergence is independent of the choice of the scalar product in $V$, but that the curl depends upon this choice and the related choice of the vector product. The chapter concludes with physical applications and the definition of harmonic vector fields.

In Chapter 9 we return to more algebra, the objective being the eventual introduction of differential forms. So here we consider tensor products, graded vector spaces, graded algebras, and exterior algebras. This is done in considerable generality, and more is included than is necessary in the sequel. This chapter, however, is a very convenient summary of this material for the expert in modern differential geometry.

Since little progress can be made without the Implicit Function Theorem (a much neglected theorem in many courses of instruction), Chapter 10 is devoted to the proof of this theorem together with the necessary topological background. The student is introduced here to the basic theorems about topological spaces and their consequences in analysis. This, of course, is essential for the serious student of mathematics, but some experience with this area of ideas is probably necessary if most students are to be successful with this chapter.

Differential forms are introduced in Chapter 11, which is devoted to their definition and basic properties. The problem of expounding this material is difficult, for it is desirable to have both the abstract approach and the specific formulas in terms of a basis. One wishes to include the algebra of differential forms, the notion of a field of forms, and the exterior derivative. For an old-time differential geometer who has been converted to the abstract approach, the reviewer believes that the presentation of this chapter is too heavily loaded with indices and coordinate transformation formulas. Anyone who has mastered the previous chapters of this book is ready for a more abstract treatment.

The purpose of Chapter 12 is to treat the integration of differential forms including the theorems of Stokes and de Rham. To start things off there is a brief discussion of differentiable singular homology so that differentiable chains are defined. This leads to integrals of forms over chains and Stokes' Theorem. It would have been helpful to many students to have treated the special case of a surface integral and Gauss' Divergence Theorem at this stage. The chapter concludes with some formulas of Green and a brief discussion of harmonic forms and cohomology.

In the final chapter, many of the preceding results for real vector 
spaces are extended to complex spaces. Here one finds the basic ideas of the calculus of complex differential forms which are used in current research on complex manifolds.

The above outline of the book raises two questions concerning the undergraduate curriculum: (1) Is this appropriate material for undergraduates? And (2), how does such a course fit in with other undergraduate courses? Let us discuss these questions simultaneously. The first five chapters are standard linear algebra which should be part of the intellectual equipment of every mathematics major. Since most institutions give such a course regularly, it seems most appropriate for this to be included there. Algebra has become so important in every branch of mathematics that such a course should be given as early as possible in the career of an undergraduate, preferably in the sophomore year and certainly no later than the junior year.

The calculus of vector-valued functions in Chapters 6-8 is an appropriate part of a second course in calculus, which will require linear algebra as a prerequisite. This seems to be a natural order of events, but undoubtedly will require considerable revision of most curricula. This course could also appropriately include the basic topology in Chapter 10.

The graded algebra in Chapter 9 is considerably more specialized and is not an essential part of the undergraduate curriculum. Most of it should be left to graduate courses for experts. A simplified approach, however, giving only the exterior algebra can be incorporated easily in a course devoted to differential forms and their applications. Such a course is a badly needed addition to the undergraduate curriculum and could well serve as part of a senior course in analysis. This would include the algebra necessary for differential forms and an improved version of Chapters 11 and 12 . The variations on the theory as it is applied to complex manifolds (Chapter 13) are probably best reserved for graduate courses.

In summary of the impact of this book on the curriculum, we see that linear algebra should be taught as early as possible, that the calculus of several variables should be based upon this algebra, and that the calculus of differential forms should be introduced as an important topic in advanced calculus.

C. B. Allendoerfer 